\title{
U-shaped Gene Induced by Ecdysone Signal Pathway in Drosophila
}

\author{
Gang Wang \\ Department of Genetics, Weifang Medical University, Weifang, 261053, China \\ Corresponding author, gangwt@126.com
}

Keywords: Drosophila; Ecdysone; U-shaped gene; Hemocyte

\begin{abstract}
The Drosophila U-shaped (ush) gene play important role in hemocyte differentiation. However, the mechanism of how the ush gene is mediated is still unclear. In this study, we reveals that ush gene expression increased during molting and metamorphosis stage of the third instar larva, 20-hydroxyecdysone (20E) treatment can up regulate ush expression, the silence of USP1 and EcR-A led to a reduced transcript level of ush gene in S2 cells. Those results indicated that the ecdysone signaling transduction pathway can mediate ush expression through its receptor.
\end{abstract}

\section{Introduction}

The insect hemocytes generation mainly happened in two periods during the development [1]. The first is embryonic period, the larval hemocytes was generated in the mesoderm [2]. The second is larval developmental stage. For now, the molecular mechanism of how insect hemocytes differentiated during embryonic period have been fully understand. Several transcription factors play important role, such as serpent (srp), lozenge (lz) and U-shaped (ush). During the larval developmental stage, the molecular mechanism of hemocytes differentiation is more complicate. The Notch, Wnt and Hedgehog $(\mathrm{HH})$ signal transduction pathway were involved in regulating these transcription factors srp, $\mathrm{lz}$ and ush, and then mediate the larval hemocytes differentiation [3].

The ush gene is member of the FOG family. Current studies have found that all FOG members function relies on the GATA transcription factor family [4]. The Drosophila ush protein contains 1191 amino acids and 9 zinc finger domain, it can interact with srpNC to regulate the fetal hemocytes differentiation [5]. During the larval stage, ush gene only express during third instar larvae [6]. After Drosophia ush gene was knocked down, the Drosophia lamellocytes number in lymph gland increased significantly, caused lymph gland enlargement, those results indicate that ush can suppress lamellocytes differentiate into other hemocytes [7]. The ush gene mainly express in drosophila plasmatocytes, and may be associated with the maintenance of plasmatocytes function [8]. However, some reseach indicated that the knock down of drosophila ush gene resulting in the prohemocytes numbers decreased, and the increased expression of ush gene suppressed crystal cells and plasmatocytes differentiation. Ush can suppressed prohemocytes differentiation and maintain the prohemocytes numbers in lymph gland [9].

For now, how ush gene is regulated during the larval hemocytes differentiation is not completely understood. In this study, we used drosophila as model animal and investigated the expression profile of ush gene utilizing real-time PCR, RNAi and hormone treatment methods. The results demonstrate that the ush gene can be induced by the ecdysone signal pathway through the the ecdysone receptor EcR-A and its heterodimer USP1. 


\section{Materials and Methods}

Insect Maintenance. The Drosophila were feed using the artificial food containing agar, glucose, antiseptic reagents, cornmeal andyeast, under the $14: 10 \mathrm{~h}$ light and dark conditions at $25 \pm 1{ }^{\circ} \mathrm{C}$.

Quantitative real-time PCR

The total RNA of drosophila and the S2 cells was isolated and was reverse transcribed into the first strand cDNA as the PCR template according to the manufacturer's instructions.

Based on the drosophila ush gene sequence, we designed the primers for the real-time PCR, ushF: 5'-gcaactatgtctccacctac-3', the reverse primer ushR: 5'-ctgcttcaccgttagataca -3' (the length is $171 \mathrm{bp}$ ). The act5C gene was designed as internal standardization with the primers act5CF: $5^{\prime}$-ctggcttcgc tgtccacctt-3' and the reverse primer act5CR 5'-gcactttcgctgctgcttcc-3'. the real-time PCR tube with $10 \mu \mathrm{l}$ volume containing TaqTM, $2 \mu \mathrm{l}$ primers $(1 \mu \mathrm{mol} / \mathrm{L})$ and $1 \mu \mathrm{l}$ cDNA (1:10 diluted $)$. The PCR was programmed: one cycle at $94{ }^{\circ} \mathrm{C}$ for $3 \mathrm{~min} ; 32$ cycles at $94^{\circ} \mathrm{C}$ for $15 \mathrm{~s}, 62{ }^{\circ} \mathrm{C}$ for $15 \mathrm{~s}$. We used the comparative $\mathrm{CT}$ method to analyze the results.

Cell Culture and Hormonal Regulation of ush gene. The S2 cells from Drosophila were cultured according to the instructions. The 20E (Sigma) which can act similar with ecdysone was diluted and added to the S2 cells, The final concentration was $1 \mu \mathrm{M} 20 \mathrm{E}$. The S2 cells were cultured for various times after 20E was added. The control cells using equal volume of DMSO. Then detected the ush gene expression levels by real-time PCR. Three independent experiments were performed and analyzed to construct a graph.

RNAi in S2 Cell Line. In order to investigate whether ecdysone receptor ECR and USP can mediate ush expression, we designed the EcR-A and USP1 gene dsRNAs using the RNAi kit (Ambion, Austin, Texas). All the primers for dsRNAs had a T7 binding sequence (5'-gcgtaatacgactcactatagg-3') on the 5' end. The EcR-A dsRNA primers, EcRiF: 5'-T7-aacaacaattagctgcctct-3', EcRiR: 5'-T7-gtccttcttgct cttcttc-3'; the USP1 dsRNA primers, USP1iF: 5'-T7-caacggttctgatgacttca-3' USP1iR: 5'-T7-gtaatgcggaaga ggaaca g-3'; the green fluorescent protein (GFP) was designed as control; the primers for GFP dsRNA was GFPiF: 5'-T7-tggtcccaattctcgtggaac-3' GFPiR: 5'-T7-cttgaagttgaccttgatgcc-3'. The dsRNA quantity was detected by $260 \mathrm{~nm}$ spectrophotometryt. The dsRNA quality was determined by $1.2 \%$ agarose electrophoresis. after the cells was transfected $10 \mathrm{~h}$, the $20 \mathrm{E}$ was added, continue culture for $6 \mathrm{~h}$, the total RNA was isolated for the real-time PCR analysis. Cells transfected with dsGFP was set as inner control. Three independent experiments were performed and analyzed to construct a graph.

\section{Results}

Ush Gene Expression Increased During Molting and Metamorphosis. In order to detect how ush gene express during the larval stage, we isolated the total RNA from the third instar at $0 \mathrm{~h}$ to 60 $\mathrm{h}$. Real-time PCR results showed that the ush mRNA level has two peaks at $0 \mathrm{~h}$ and $60 \mathrm{~h}$ when the larvae body underwent molting and metamorphosis (Fig. 1).

20E Upregulated the Expression of Pvr in S2 Cells. In order to detect how the 20E affect the expression of ush, we used 20E treat the $\mathrm{S} 2$ cells for $1 \mathrm{~h}, 3 \mathrm{~h}, 6 \mathrm{~h}$ and $12 \mathrm{~h}$ respectively. Then total RNA was isolated and real-time PCR was performed to detect the ush gene expression profile. Results showed that the ush mRNA level began to increase from $3 \mathrm{~h}$ until $6 \mathrm{~h}$ after $20 \mathrm{E}$ treatment, then the mRNA level dramatically decreased at $12 \mathrm{~h}$ (Fig. 2). Those results indicated that ush gene can be induced by insect ecdysone. 
Silence of EcR-A and USP1 Blocking the Expression of Pvr. In order to determine how the ecdysone receptors EcR and USP1 affect the ush gene expression, EcR-A and USP1 were silenced using RNAi method in S2 cells. Real-time PCR results indicated that the silence of EcR-A and USP1 lead to the significant suppression of ush gene induced by $20 \mathrm{E}$ compared to the control transfected with dsGFP. This results certified that the ush gene involved in the ecdysone signal pathway and it acts downstream of the ecdysone receptors (Fig. 3).
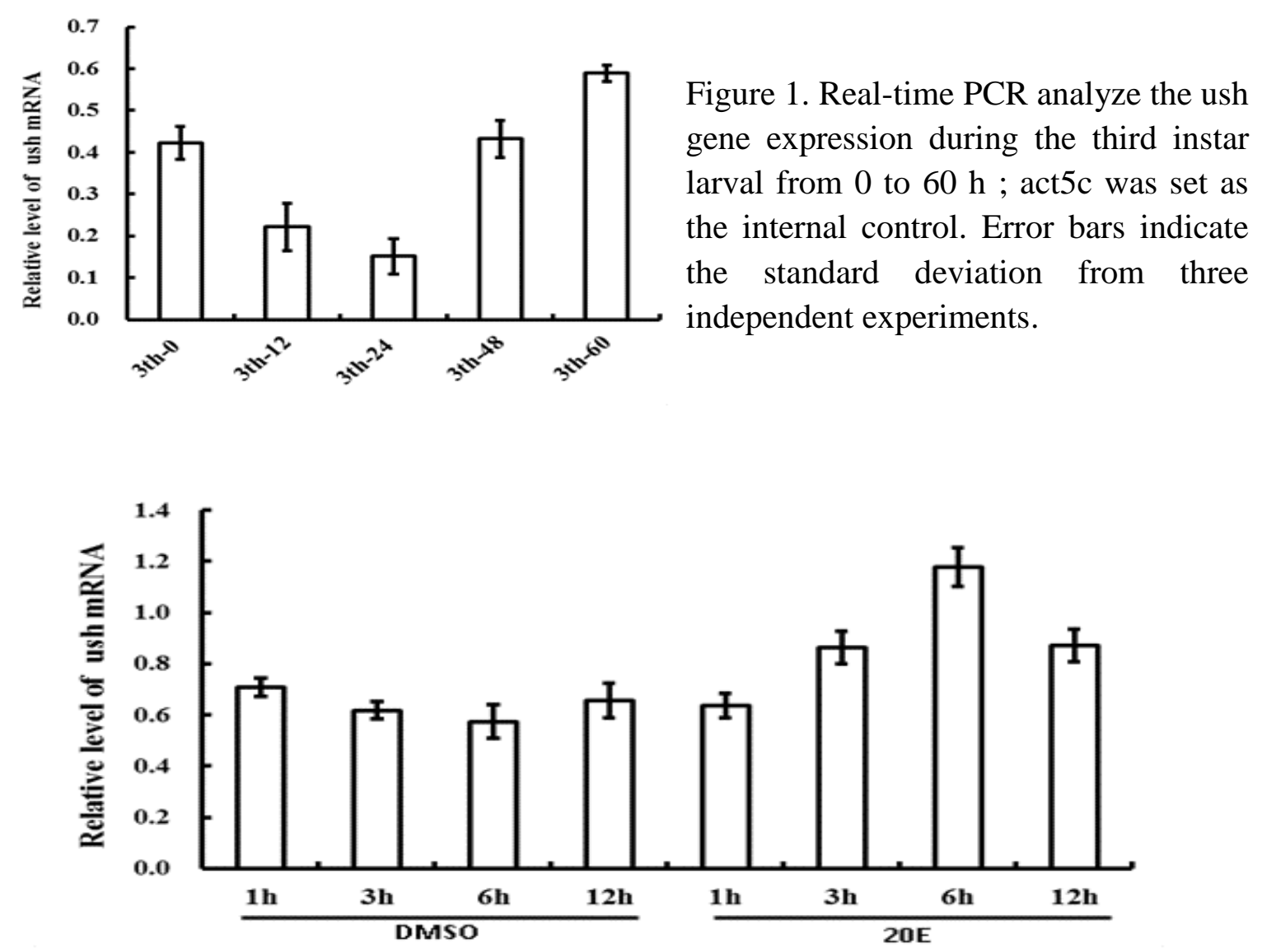

Figure 2. Real-time PCR analyze the ush gene expression from S2 cells after 20E treatment. The ush expression was measured at 1,3,6, and $12 \mathrm{~h}$ after hormonal treatment. Control was treated equal dilution of DMSO. Error bars indicate the standard deviation from three independent experiments.

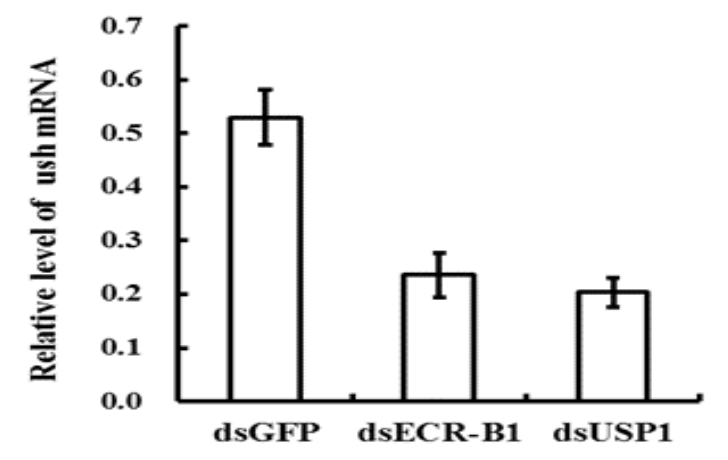

Figure 3. Real-time PCR analyze the ush gene expression from $\mathrm{S} 2$ cells after EcR-A and USP1 were silenced. Error bars indicate the standard deviation from three independent experiments. 


\section{Discussion}

In this study, we take drosophila as model animal and used quantitative real-time PCR and RNAi methods to investigate the Drosophila ush gene expression profile. The results showed that the ush gene expression increased during insect molting and metamorphosis stage, ecdysone can induce ush gene abundantly expressed, RNAi research results showed that the silenced of EcR-A and USP1 can block the ush gene expression. Based on those results, we can conclude that drosophila ush gene involved in the ecdysone signal transduction pathway and it can be induced by ecdysone through ecdysone receptor EcR and USP.

Insect larvae hemocytes differentiation is a complex process, this process is not only mediated by some transcription factors, such as srp, lz and ush, but also regulated by Notch, Wnt and Hedgehog $(\mathrm{HH})$ signaling pathway [10-13]. The ush gene belong to FOG family, the current studies found that all the FOG members function depends on the GATA transcription factors [3]. The ush can also interact with the srp to mediate the insect hemocytes differentiation $[14,15]$. In this study, we found that the ush gene not only express during molting and metamorphosis when hemocytes undergo differentiation, but the ush gene also express during feed stage. It is unclear what roles the ush play during the feed stage when hemocytes not undergo differentiation. Drosophila GATA transcription factors has five isomers, whether ush play roles through acting with other isomers need further investigate.

Although the specific role of ush on larvae hemocytes differentiation is still unclear, but the knockdown of ush gene resulting in the destruction of drosophila hemocytes differentiation [15]. This results suggest that ush play an important role in larval hemocytes development. Our study founded that $20 \mathrm{E}$ can induce the expression of ush through ecdysone receptor, this suggest that the ush is a downstream member in the ecdysone signal transduction pathway, ecdysone may mediate larval hemocytes differentiation through up regulating ush gene.

\section{Acknowledgements}

This research was supported by the Scientific Funds for Outstanding Young Scientists of Shandong Province Award [BS2013SW030], the Project of the Shandong Province Higher Educational Science and Technology Program (J12LK01) and the Natural Science Foundation of Shandong Province (ZR2012HL42)

\section{References}

[1] M. Crozatier and M. Meister, "Drosophila haematopoiesis," Cell Microbiol, vol. 9, pp. 1117-26, May 2007.

[2] A. Holz, B. Bossinger, T. Strasser, W. Janning, and R. Klapper, "The two origins of hemocytes in Drosophila," Development, vol. 130, pp. 4955-62, Oct 2003.

[3] L. Waltzer, V. Gobert, D. Osman, and M. Haenlin, "Transcription factor interplay during Drosophila haematopoiesis," Int J Dev Biol, vol. 54, pp. 1107-15, 2010.

[4] L. Pang, H. H. Xue, G. Szalai, X. Wang, Y. Wang, D. K. Watson, et al., "Maturation stage-specific regulation of megakaryopoiesis by pointed-domain Ets proteins," Blood, vol. 108, pp. 2198-206, Oct 12006. 
[5] Y. Cubadda, P. Heitzler, R. P. Ray, M. Bourouis, P. Ramain, W. Gelbart, et al., "u-shaped encodes a zinc finger protein that regulates the proneural genes achaete and scute during the formation of bristles in Drosophila," Genes Dev, vol. 11, pp. 3083-95, Nov 151997.

[6] H. Gao, X. Wu, and N. Fossett, "Upregulation of the Drosophila Friend of GATA gene U-shaped by JAK/STAT signaling maintains lymph gland prohemocyte potency," Mol Cell Biol, vol. 29, pp. 6086-96, Nov 2009.

[7] R. P. Sorrentino, T. Tokusumi, and R. A. Schulz, "The Friend of GATA protein U-shaped functions as a hematopoietic tumor suppressor in Drosophila," Developmental Biology, vol. 311, pp. 311-323, Nov 152007.

[8] L. Waltzer, L. Bataille, S. Peyrefitte, and M. Haenlin, "Two isoforms of Serpent containing either one or two GATA zinc fingers have different roles in Drosophila haematopoiesis," Embo Journal, vol. 21, pp. 5477-5486, Oct 152002.

[9] H. J. Gao, X. R. Wu, and N. Fossett, "Upregulation of the Drosophila Friend of GATA Gene u-shaped by JAK/STAT Signaling Maintains Lymph Gland Prohemocyte Potency," Molecular and Cellular Biology, vol. 29, pp. 6086-6096, Nov 152009.

[10]S. Chiba, "Notch signaling in stem cell systems," Stem Cells, vol. 24, pp. 2437-47, Nov 2006.

[11] V. Hartenstein, "Blood cells and blood cell development in the animal kingdom," Annu Rev Cell Dev Biol, vol. 22, pp. 677-712, 2006.

[12]A. P. McMahon, P. W. Ingham, and C. J. Tabin, "Developmental roles and clinical significance of hedgehog signaling," Curr Top Dev Biol, vol. 53, pp. 1-114, 2003.

[13]T. Reya and H. Clevers, "Wnt signalling in stem cells and cancer," Nature, vol. 434, pp. 843-50, Apr 142005.

[14]S. Muratoglu, B. Hough, S. T. Mon, and N. Fossett, "The GATA factor Serpent cross-regulates lozenge and u-shaped expression during Drosophila blood cell development," Developmental Biology, vol. 311, pp. 636-649, Nov 152007.

[15]R. P. Sorrentino, T. Tokusumi, and R. A. Schulz, "The Friend of GATA protein U-shaped functions as a hematopoietic tumor suppressor in Drosophila," Dev Biol, vol. 311, pp. 311-23, Nov 152007. 